\title{
Deterministic photonic spatial-polarization hyper-controlled-not gate assisted by quantum dot inside one-side optical microcavity *
}

\author{
Bao-Cang Ren, Hai-Rui Wei and Fu-Guo Deng ${ }^{\dagger}$ \\ Department of Physics, Applied Optics Beijing Area Major Laboratory, \\ Beijing Normal University, Beijing 100875, China
}

(Dated: October 3, 2018)

\begin{abstract}
Up to now, all the works about constructing quantum logic gates, an essential part in quantum computing, are focused on operating on one degree of freedom (DOF) of quantum systems. Here, we investigate the possibility to achieve a scalable photonic quantum computing based on two DOFs of quantum systems and construct a deterministic hyper-controlled-not (hyper-CNOT) gate operating on both spatial-mode and polarization DOFs of a photon pair simultaneously, by using the giant optical Faraday rotation induced by a single-electron spin in a quantum dot inside a one-side optical microcavity as a result of cavity quantum electrodynamics. With this hyper-CNOT gate and linear optical elements, two-photon four-qubit cluster entangled states can be prepared and analyzed, which gives an application to manipulate more information with less resources. We analyze the experimental feasibility of this hyper-CNOT gate and show that it can be implemented with current technology.
\end{abstract}

Keywords: Spatial-polarization hyper-controlled-not gate, photonic quantum computing, deterministic, quantum dot, one-side optical microcavity

PACS numbers: 03.67.Lx, 42.50.Ex, 42.50.Pq, 78.67.Hc

\section{Introduction}

Quantum mechanics theory, the core of quantum information, can largely improve the power of dealing and transmitting information. Quantum computing [1] is required for the precise control and manipulation of the states of quantum systems in quantum information process. It is proven that two-qubit controlled-not (CNOT) gates (or the equivalent two-qubit quantum gates) assisted by single-qubit gates are sufficient for universal quantum computing, so it is important to construct a two-qubit CNOT gate. By far, many works have been done on constructing a two-qubit CNOT gate or its equivalent in notably trapped ions [2], nuclear magnetic spins [3], free electrons [4], and polarized photons [5-10]. The pioneering work by Knill, Laflamme, and Milburn [5] showed that a photonic CNOT gate could be created with the maximal success probability of $3 / 4$, resorting to only single-photon sources, detectors, and linear optical elements such as beam splitters. Since this original work, there has been a significant progress [6 9] in constructing a CNOT gate with linear optics, which realizes probabilistically the nonlinear coupling between two photons by using interference, at least two ancilla photons, single-photon detectors, and conditioning. With nonlinear optics, a deterministic CNOT gate can also be constructed. In 2004, Nemoto and Munro [10] constructed a near deterministic CNOT gate using several single photon sources, linear-optical elements, photon

\footnotetext{
* Published in Laser Phys. Lett. 10 (2013) 095202

${ }^{\dagger}$ Corresponding author: fgdeng@bnu.edu.cn
}

number resolving quantum nondemolition detectors, and feed-forward. The key element of the CNOT gate in their proposal is the quantum nondemolition detector (QND) based on cross-Kerr nonlinearity.

Although there are many valuable works on constructing quantum logical gates, especially CNOT gates, they are all focused on operating on one degree of freedom (DOF) of quantum systems. There are, by far, no quantum entangling gates operating on more than one DOF of quantum systems. In fact, there are many advantages for dealing with quantum information process in a larger Hilbert space, especially for its robustness against noise [11] and the high channel capacity. High-dimensional entanglement has been realized in multipartite [12, 13] and multidimensional [14, 15] quantum systems. Although a universal quantum computing can be realized with twoqubit CNOT gates and single-qubit operations, it will be convenient to have multi-qubit quantum logic gates in quantum computation. In 2006, Fiurášek [16] proposed some schemes for the probabilistic direct realization of the fundamental Toffoli and Fredkin gates with linear optics, and he presented a scheme for linear optical quantum Fredkin gate based on the combination of experimentally demonstrated linear optical partial-SWAP gate and controlled-Z gates in 2008 [17]. Gong et al [18] also discussed the realization of a quantum Fredkin gate by using CNOT gates with only linear optics and single photons. The large Hilbert space with more than one DOF has also been discussed in some applications in quantum communication in the past few years [19 24], especially for hyperentanglement which is defined as quantum systems entangled in more than one DOF. Besides the task in which hyperentanglement is used to assist quantum information processing in one DOF, the complete ana- 
lyzer for hyperentangled Bell states has also been constructed [25] with cross-kerr nonlinearity to increase the channel capacity of long-distance quantum communication in more than one DOF.

In this Letter, we investigate the possibility of achieving scalable photonic quantum computing based on two DOFs of quantum systems, which is different from all the existing works about constructing quantum logic gates operating on one DOF of quantum systems. We construct a deterministic hyper-controlled-not (hyperCNOT) gate which is used to perform a CNOT gate on both the spatial-mode and the polarization DOFs of a two-photon system simultaneously, assisted by two quantum dots embodied in one-side optical microcavities (QD-cavity). Exploiting the giant optical Faraday rotation of the left-circularly and the right-circularly polarized photons induced by a single-electron spin in a QD-cavity system, we construct a four-qubit controlled$\mathrm{Z}$ (CZ) gate by using the two electron spins as the control qubits and the polarization and the spatial mode of a photon as the two target qubits, respectively. After the second photon interacts with the two QD-cavity systems, the two electron spins are detected, and a deterministic hyper-CNOT gate could be constructed with feed-forward single-qubit operations. As an application of this hyper-CNOT gate, one can use it to prepare entangled two-photon four-qubit cluster states easily and analyze the 16 hyperentangled cluster states simply, resorting to some linear optical elements. We analyze the experimental feasibility of this hyper-CNOT gate, and our result shows that it can be implemented with current technology.

\section{Construction of deterministic spatial-polarization hyper-controlled-not gate}

In 2008, Hu et al [26, 27] pointed out that the interaction of left-circularly and right-circularly polarized photons with a QD-cavity system can be used in quantum information process. With this optical property of QDcavity systems, multi-qubit entangler [26 28] and photonic polarization Bell-state analyzer [29, 30] can be constructed. In 2010, Bonato et al 30] constructed a CNOT gate operating on hybrid quantum system composed of a photon and an electron spin using the interface between the photon and the electron spin in a double-sided QDcavity system in the weak coupling regime.

The QD-cavity system used in our proposal is constructed by a singly charged QD (e.g., a self-assembled $\mathrm{In}(\mathrm{Ga})$ As QD or a GaAs interface QD) located in the center of a one-side optical resonant cavity (the bottom distributed Bragg reflectors is 100\% reflective and the top distributed Bragg reflectors is partially reflective) to achieve the maximal light-matter coupling. According to Pauli's exclusion principle, a negatively charged exciton $\left(X^{-}\right)$consisted of two electrons bound to one hole [31] can be optically excited when an excess electron is in- jected into the $\mathrm{QD}$. The optical resonance of $X^{-}$with circularly polarized photons depends on the excess electron spin in the QD [32], shown in Fig 1, For the excess electron-spin state $|\uparrow\rangle$, the negatively charged exciton $|\uparrow \downarrow \uparrow\rangle$ with two electron spins antiparallel is created by resonantly absorbing a left-circularly polarized photon $|L\rangle$. Here $|\Uparrow\rangle$ describes the heavy-hole spin state $\left|+\frac{3}{2}\right\rangle$. For the excess electron spin $|\downarrow\rangle$, the other negatively charged exciton $|\downarrow \uparrow \downarrow\rangle$ can be created by resonantly absorbing a right-circularly polarized photon $|R\rangle$. Here $|\Downarrow\rangle$ describes the heavy-hole spin state $\left|-\frac{3}{2}\right\rangle$. This optical process can be described by Heisenberg equations for the cavity field operator $\hat{a}$ and $X^{-}$dipole operator $\hat{\sigma}_{-}$in the interaction picture [33],

$$
\begin{aligned}
& \frac{d \hat{a}}{d t}=-\left[i\left(\omega_{c}-\omega\right)+\frac{\kappa}{2}+\frac{\kappa_{s}}{2}\right] \hat{a}-g \hat{\sigma}_{-}-\sqrt{\kappa} \hat{a}_{i n}, \\
& \frac{d \hat{\sigma}_{-}}{d t}=-\left[i\left(\omega_{X^{-}}-\omega\right)+\frac{\gamma}{2}\right] \hat{\sigma}_{-}-g \hat{\sigma}_{z} \hat{a}, \\
& \hat{a}_{\text {out }}=\hat{a}_{\text {in }}+\sqrt{\kappa} \hat{a},
\end{aligned}
$$

where $g$ represents the coupling strength between the cavity mode and $X^{-} . \kappa / 2$ and $\kappa_{s} / 2$ represent the decay rate and the side leakage rate of the cavity field, respectively. $\gamma / 2$ represents the decay rate of $X^{-} . \omega_{X^{-}}, \omega$, and $\omega_{c}$ represent the frequencies of the $X^{-}$transition, the input probe light, and the cavity mode, respectively.

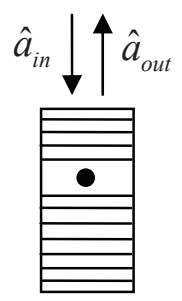

(a)

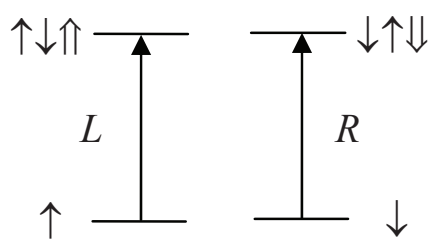

(b)
FIG. 1: The $X^{-}$spin-dependent transitions with circularly polarized photons. (a) A charged QD inside a one-side micropillar microcavity interacting with circularly polarized photons. (b) $X^{-}$spin-dependent optical transition rules due to the Pauli's exclusion principle. $L$ and $R$ represent a leftcircularly and a right-circularly polarized photons, respectively. $\uparrow$ and $\downarrow$ represent the spins of the excess electron. $\uparrow \downarrow \uparrow$ and $\downarrow \uparrow \Downarrow$ represent negatively charged exciton $X^{-}$.

If $X^{-}$stays in the ground state at the most time (i.e., $\left\langle\sigma_{z}\right\rangle=-1$ ), the reflection coefficient of a QD-cavity system for this weak excitation condition is [26]

$r(\omega)=1-\frac{\kappa\left[i\left(\omega_{X^{-}}-\omega\right)+\frac{\gamma}{2}\right]}{\left[i\left(\omega_{X^{-}}-\omega\right)+\frac{\gamma}{2}\right]\left[i\left(\omega_{c}-\omega\right)+\frac{\kappa}{2}+\frac{\kappa_{s}}{2}\right]+g^{2}}$.

In the condition $g=0$, the QD is uncoupled to the cavity (the cold cavity), and the reflection coefficient becomes

$$
r_{0}(\omega)=\frac{i\left(\omega_{c}-\omega\right)-\frac{\kappa}{2}+\frac{\kappa_{s}}{2}}{i\left(\omega_{c}-\omega\right)+\frac{\kappa}{2}+\frac{\kappa_{s}}{2}}
$$


Now, the optical process based on the $X^{-}$spindependent transitions is obtained. For a light in the state $|L\rangle$, a phase shift of $\varphi_{h}$ is gotten for a hot cavity (the QD is coupled to the cavity) with the excess electron spin in the state $|\uparrow\rangle$, and a phase shift of $\varphi_{0}$ is gotten for a cold cavity with the excess electron spin in the state $|\downarrow\rangle$. For a light in the state $|R\rangle$, a phase shift of $\varphi_{0}$ is gotten for a cold cavity with the excess electron spin in the state $|\uparrow\rangle$, and a phase shift of $\varphi_{h}$ is gotten for a hot cavity with the excess electron spin in the state $|\downarrow\rangle$. By adjusting the frequencies of the light $(\omega)$ and the cavity mode $\left(\omega_{c}\right)$, the reflection coefficient can reach $\left|r_{0}(\omega)\right| \cong 1$ and $\left|r_{h}(\omega)\right| \cong 1$ when the cavity side leakage is negligible. If the linearly polarized probe beam in the state $\frac{1}{\sqrt{2}}(|R\rangle+|L\rangle)$ is put into a one-side QD-cavity system in the superposition spin state $\frac{1}{\sqrt{2}}(|\uparrow\rangle+|\downarrow\rangle)$, the state of system composed by the light and the electron spin after reflection is

$$
\begin{aligned}
& \frac{1}{2}(|R\rangle+|L\rangle) \otimes(|\uparrow\rangle+|\downarrow\rangle) \rightarrow \frac{1}{2} e^{i \varphi_{0}}\left[\left(|R\rangle+e^{i \Delta \varphi}|L\rangle\right)\right. \\
&\left.|\uparrow\rangle+\left(e^{i \Delta \varphi}|R\rangle+|L\rangle\right)|\downarrow\rangle\right],
\end{aligned}
$$

where $\Delta \varphi=\varphi_{h}-\varphi_{0}, \varphi_{0}=\arg \left[r_{0}(\omega)\right]$, and $\varphi_{h}=$ $\arg \left[r_{h}(\omega)\right] \cdot \theta_{F}^{\uparrow}=\left(\varphi_{0}-\varphi_{h}\right) / 2=-\theta_{F}^{\downarrow}$ is a Faraday rotation angle. After reflection, the light and the spin become entangled with the different phase shifts of $|L\rangle$ and $|R\rangle$ photons.

The principle of our deterministic spatial-polarization hyper-CNOT gate for a two-photon system in two DOFs with the optical property of one-side QD-cavity systems is shown in Fig 2] which can be used to perform a CNOT gate operation on both the spatial mode and the polarization DOFs of a two-photon system by flipping the spatialmode target qubit and the polarization target qubit if the two control qubits are in the spatia mode $\left|i_{2}\right\rangle$ and in the polarization state $|L\rangle$, respectively. The $\mathrm{QD}_{1}$ and $\mathrm{QD}_{2}$ are used to operate on the spatial-mode and the polarization DOFs, respectively. By adjusting the frequencies $\omega-\omega_{c} \approx \kappa / 2$ to get the phase shifts of the left and the right circularly polarized photons as $\varphi_{0}=-\pi / 2$ and $\varphi_{h}=0$, the interaction of a single photon with a QD-cavity system can be described as

$$
\begin{aligned}
|R, \uparrow\rangle & \rightarrow-i|R, \uparrow\rangle, & |L, \uparrow\rangle & \rightarrow|L, \uparrow\rangle, \\
|R, \downarrow\rangle & \rightarrow|R, \downarrow\rangle, & |L, \downarrow\rangle & \rightarrow-i|L, \downarrow\rangle .
\end{aligned}
$$

\section{(1) Three-particle four-qubit hybrid controlled-Z gate}

We assume the initial states of the excess electron spin in $\mathrm{QD}_{1}$ and the photon $a$ are $\frac{1}{\sqrt{2}}(i|\uparrow\rangle+|\downarrow\rangle)_{e_{1}}$ and $\left|\Phi_{a}\right\rangle_{0} \equiv\left(\alpha_{1}|R\rangle+\alpha_{2}|L\rangle\right)_{a} \otimes\left(\gamma_{1}\left|a_{1}\right\rangle+\gamma_{2}\left|a_{2}\right\rangle\right)$, respectively. Here $\left|a_{1}\right\rangle$ and $\left|a_{2}\right\rangle$ represent the two spatial modes of the photon $a$. After the photon $a$ passes through the circular polarizing beam splitter CPBS $\left(\mathrm{CPBS}_{1}\right.$ and $\left.\mathrm{CPBS}_{3}\right)$ and the half-wave plate $\mathrm{HWP}_{1}\left(\mathrm{X}_{1}\right.$ and $\left.\mathrm{X}_{3}\right)$, interacts with the electron spin in $\mathrm{QD}_{1}$, and then passes through another $\mathrm{HWP}_{1}\left(\mathrm{X}_{2}\right.$ and $\left.\mathrm{X}_{4}\right)$, CPBS $\left(\mathrm{CPBS}_{2}\right.$ and $\left.\mathrm{CPBS}_{4}\right)$

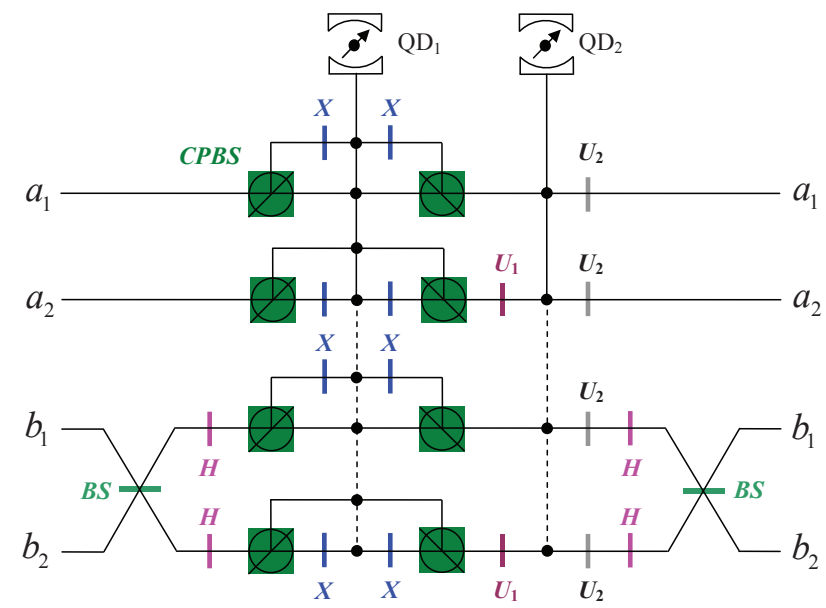

FIG. 2: (Color online) Schematic diagram of our spatialpolarization hyper-controlled-not gate operating on both the spatial-mode and polarization degrees of freedom of a twophoton system simultaneously. BS represents a 50:50 beam splitter which is used to perform a Hadamard operation on the spatial-mode states of a photon. CPBS represents a polarizing beam splitter in the circular basis, which transmits the photon in the right-circular polarization $|R\rangle$ and reflects the photon in the left-circular polarization $|L\rangle$, respectively. $X_{j}$ represents a half-wave plate $\left(\mathrm{HWP}_{1}\right)$ which is used to perform a polarization bit-flip operation $X_{j}=|R\rangle\langle L|+| L\rangle\langle R|$ $(j=1,2, \ldots, 8) . \quad H_{k}$ represents a half-wave plate $\left(\mathrm{HWP}_{2}\right)$ which is used to perform a polarization Hadamard operation $\left[|R\rangle \leftrightarrow \frac{1}{\sqrt{2}}(|R\rangle+|L\rangle)\right.$ and $\left.|L\rangle \leftrightarrow \frac{1}{\sqrt{2}}(|R\rangle-|L\rangle)\right](k=1,2,3,4)$. $U_{1 m}$ represents a wave plate $\left(\mathrm{WP}_{1}\right)$ which is used to perform a polarization phase-flip operation $U_{1 m}=-i|R\rangle\langle R|-i| L\rangle\langle L|$ to achieve the relative phase shift $-i$ between the two spatial modes of a photon $(m=2,4)$. $U_{2 n}$ represents a wave plate $\left(\mathrm{WP}_{2}\right)$ which is used to perform a polarization phase-flip operation $U_{2 n}=|R\rangle\langle R|-i| L\rangle\langle L|(n=1,2,3,4)$.

and the wave plate $\mathrm{WP}_{1}\left(\mathrm{U}_{12}\right)$ in Fig 2, the state of the system composed of $\mathrm{QD}_{1}$ and the photon $a$ is changed from $\left|\Phi_{a e_{1}}\right\rangle_{0}$ to $\left|\Phi_{a e_{1}}\right\rangle_{1}$. Here

$$
\begin{aligned}
\left|\Phi_{a e_{1}}\right\rangle_{0}= & \left(\alpha_{1}|R\rangle+\alpha_{2}|L\rangle\right)_{a} \otimes\left(\gamma_{1}\left|a_{1}\right\rangle+\gamma_{2}\left|a_{2}\right\rangle\right) \\
\otimes & \frac{1}{\sqrt{2}}(i|\uparrow\rangle+|\downarrow\rangle)_{e_{1}}, \\
\left|\Phi_{a e_{1}}\right\rangle_{1}= & \frac{1}{\sqrt{2}}\left(\alpha_{1}|R\rangle+\alpha_{2}|L\rangle\right)_{a} \otimes\left[| \uparrow \rangle _ { e _ { 1 } } \left(\gamma_{1}\left|a_{1}\right\rangle\right.\right. \\
& \left.\left.\quad+\gamma_{2}\left|a_{2}\right\rangle\right)+|\downarrow\rangle_{e_{1}}\left(\gamma_{1}\left|a_{1}\right\rangle-\gamma_{2}\left|a_{2}\right\rangle\right)\right] .
\end{aligned}
$$

This is the result of the controlled- $Z$ gate constructed by using the electron spin $e_{1}$ as the control qubit and the spatial modes of the photon $a$ as the target qubit.

The initial state of $\mathrm{QD}_{2}$ in Fig 2 is prepared to be $\frac{1}{\sqrt{2}}(i|\uparrow\rangle+|\downarrow\rangle)_{e_{2}}$, and the frequencies of the input photon and the cavity mode are adjusted to be $\omega-\omega_{c} \approx \kappa / 2$, as the same as those for $\mathrm{QD}_{1}$. After the photon $a$ interacts with $\mathrm{QD}_{2}$ and passes through $\mathrm{WP}_{2}\left(\mathrm{U}_{21}\right.$ and $\left.\mathrm{U}_{22}\right)$ as shown in Fig 2, the state of the complicated system composed of $\mathrm{QD}_{1}, \mathrm{QD}_{2}$, and the photon $a$ is changed 
from $\left|\Phi_{a e_{1} e_{2}}\right\rangle_{1}$ to $\left|\Phi_{a e_{1} e_{2}}\right\rangle_{2}$. Here

$$
\begin{aligned}
\left|\Phi_{a e_{1} e_{2}}\right\rangle_{1}= & \left|\Phi_{a e_{1}}\right\rangle_{1} \otimes \frac{1}{\sqrt{2}}(i|\uparrow\rangle+|\downarrow\rangle)_{e_{2}} \\
\left|\Phi_{a e_{1} e_{2}}\right\rangle_{2}= & \frac{1}{2}\left[|\uparrow\rangle_{e_{2}}\left(\alpha_{1}|R\rangle+\alpha_{2}|L\rangle\right)_{a}+|\downarrow\rangle_{e_{2}}\left(\alpha_{1}|R\rangle\right.\right. \\
& \left.\left.-\alpha_{2}|L\rangle\right)_{a}\right] \otimes\left[|\uparrow\rangle_{e_{1}}\left(\gamma_{1}\left|a_{1}\right\rangle+\gamma_{2}\left|a_{2}\right\rangle\right)\right. \\
& \left.+|\downarrow\rangle_{e_{1}}\left(\gamma_{1}\left|a_{1}\right\rangle-\gamma_{2}\left|a_{2}\right\rangle\right)\right]
\end{aligned}
$$

This is the result of the three-particle four-qubit hybrid controlled-Z gate constructed by using the two electron spins as the control qubits and the polarization and the spatial modes of the photon $a$ as the two target qubits. That is, when the spin of the electron $e_{1}$ is in the state $|\downarrow\rangle_{e_{1}}$, the spatial mode $\left|a_{2}\right\rangle$ of the photon $a$ obtains a phase shift $\pi$, and when the spin of the electron $e_{2}$ is in the state $|\downarrow\rangle_{e_{2}}$, the polarization mode $|L\rangle$ of the photon $a$ obtains a phase shift $\pi$.

\section{(2) Spatial-polarization hyper-CNOT gate}

The second photon $b$ is prepared initially in the state $\left|\Phi_{b}\right\rangle_{0}=\left(\beta_{1}|R\rangle+\beta_{2}|L\rangle\right)_{b} \otimes\left(\delta_{1}\left|b_{1}\right\rangle+\delta_{2}\left|b_{2}\right\rangle\right)$. After Hadamard operations are performed on both the spatial mode and the polarization DOFs of the photon $b$ (by making the photon $b$ pass through $B S_{1}, H_{1}$ and $\left.H_{2}\right)$, the state $\left|\Phi_{b}\right\rangle_{0}$ is changed to be $\left|\Phi_{b}^{\prime}\right\rangle_{0} \equiv\left(\beta_{1}^{\prime}|R\rangle+\right.$ $\left.\beta_{2}^{\prime}|L\rangle\right)_{b} \otimes\left(\delta_{1}^{\prime}\left|b_{1}\right\rangle+\delta_{2}^{\prime}\left|b_{2}\right\rangle\right)$. Here $\beta_{1}^{\prime}=\frac{1}{\sqrt{2}}\left(\beta_{1}+\beta_{2}\right)$, $\beta_{2}^{\prime}=\frac{1}{\sqrt{2}}\left(\beta_{1}-\beta_{2}\right), \delta_{1}^{\prime}=\frac{1}{\sqrt{2}}\left(\delta_{1}+\delta_{2}\right)$ and $\delta_{2}^{\prime}=\frac{1}{\sqrt{2}}\left(\delta_{1}-\delta_{2}\right)$. Then we perform unitary operations on the two electron spins in $\mathrm{QD}_{1}$ and $\mathrm{QD}_{2}$, which transform the states $|\uparrow\rangle$ and $|\downarrow\rangle$ to $\frac{1}{\sqrt{2}}(i|\uparrow\rangle+|\downarrow\rangle)$ and $\frac{1}{\sqrt{2}}(i|\uparrow\rangle-|\downarrow\rangle)$, respectively. Subsequently, we let the photon $b$ pass through CPBS $\left(\mathrm{CPBS}_{5}\right.$ and $\left.\mathrm{CPBS}_{7}\right), \mathrm{HWP}_{1}\left(\mathrm{X}_{5}\right.$ and $\left.\mathrm{X}_{7}\right), \mathrm{QD}_{1}$, $\mathrm{HWP}_{1}\left(\mathrm{X}_{6}\right.$ and $\left.\mathrm{X}_{8}\right), \mathrm{CPBS}\left(\mathrm{CPBS}_{6}\right.$ and $\left.\mathrm{CPBS}_{8}\right), \mathrm{WP}_{1}$ $\left(\mathrm{U}_{14}\right), \mathrm{QD}_{2}$ and $\mathrm{WP}_{2}\left(\mathrm{U}_{23}\right.$ and $\left.\mathrm{U}_{24}\right)$. After the interaction between the photon $b$ and the two QDs, the state of system composed of $\mathrm{QD}_{1}, \mathrm{QD}_{2}$, and the photons $a$ and $b$ is changed from $\left|\Psi_{s}\right\rangle_{1}$ to $\left|\Psi_{s}\right\rangle_{2}$. Here

$$
\begin{aligned}
\left|\Psi_{s}\right\rangle_{1}= & \left|\Phi_{a e_{1} e_{2}}\right\rangle_{2} \otimes\left(\beta_{1}^{\prime}|R\rangle+\beta_{2}^{\prime}|L\rangle\right)_{b}\left(\delta_{1}^{\prime}\left|b_{1}\right\rangle+\delta_{2}^{\prime}\left|b_{2}\right\rangle\right) \\
\left|\Psi_{s}\right\rangle_{2}= & {\left[|\uparrow\rangle_{e_{1}} \gamma_{1}\left|a_{1}\right\rangle\left(\delta_{1}^{\prime}\left|b_{1}\right\rangle+\delta_{2}^{\prime}\left|b_{2}\right\rangle\right)+|\downarrow\rangle_{e_{1}} \gamma_{2}\left|a_{2}\right\rangle\right.} \\
& \left.\times\left(\delta_{1}^{\prime}\left|b_{1}\right\rangle-\delta_{2}^{\prime}\left|b_{2}\right\rangle\right)\right] \otimes\left[| \uparrow \rangle _ { e _ { 2 } } \alpha _ { 1 } | R \rangle _ { a } \left(\beta_{1}^{\prime}|R\rangle\right.\right. \\
& \left.\left.+\beta_{2}^{\prime}|L\rangle\right)_{b}+|\downarrow\rangle_{e_{2}} \alpha_{2}|L\rangle_{a}\left(\beta_{1}^{\prime}|R\rangle-\beta_{2}^{\prime}|L\rangle\right)_{b}\right] .
\end{aligned}
$$

By performing Hadamard operations on the two electron spins in $\mathrm{QD}_{1}$ and $\mathrm{QD}_{2}$ again after the photon $b$ passes through $\mathrm{WP}_{2}\left(\mathrm{U}_{23}\right.$ and $\left.\mathrm{U}_{24}\right)$, the state of the complicated system becomes

$$
\begin{aligned}
\left|\Psi_{s}\right\rangle_{3}= & \frac{1}{2}\left\{| \uparrow \rangle _ { e _ { 1 } } \left[\gamma_{1}\left|a_{1}\right\rangle\left(\delta_{1}^{\prime}\left|b_{1}\right\rangle+\delta_{2}^{\prime}\left|b_{2}\right\rangle\right)+\gamma_{2}\left|a_{2}\right\rangle\left(\delta_{1}^{\prime}\left|b_{1}\right\rangle\right.\right.\right. \\
& \left.\left.-\delta_{2}^{\prime}\left|b_{2}\right\rangle\right)\right]+|\downarrow\rangle_{e_{1}}\left[\gamma_{1}\left|a_{1}\right\rangle\left(\delta_{1}^{\prime}\left|b_{1}\right\rangle+\delta_{2}^{\prime}\left|b_{2}\right\rangle\right)\right. \\
& \left.\left.-\gamma_{2}\left|a_{2}\right\rangle\left(\delta_{1}^{\prime}\left|b_{1}\right\rangle-\delta_{2}^{\prime}\left|b_{2}\right\rangle\right)\right]\right\} \\
& \otimes\left\{| \uparrow \rangle _ { e _ { 2 } } \left[\alpha_{1}|R\rangle_{a}\left(\beta_{1}^{\prime}|R\rangle+\beta_{2}^{\prime}|L\rangle\right)_{b}+\alpha_{2}|L\rangle_{a}\right.\right. \\
& \left.\left(\beta_{1}^{\prime}|R\rangle-\beta_{2}^{\prime}|L\rangle\right)_{b}\right]+|\downarrow\rangle_{2}\left[\alpha _ { 1 } | R \rangle _ { a } \left(\beta_{1}^{\prime}|R\rangle\right.\right. \\
& \left.\left.\left.+\beta_{2}^{\prime}|L\rangle\right)_{b}-\alpha_{2}|L\rangle_{a}\left(\beta_{1}^{\prime}|R\rangle-\beta_{2}^{\prime}|L\rangle\right)_{b}\right]\right\} .
\end{aligned}
$$

At last, we perform Hadamard operations on both the spatial-mode $\left(\mathrm{BS}_{2}\right)$ and the polarization $\left(\mathrm{H}_{3}\right.$ and $\left.\mathrm{H}_{4}\right)$ DOFs of the photon $b$, the state $\left|\Psi_{s}\right\rangle_{3}$ is transformed into

$$
\begin{aligned}
\left|\Psi_{s}\right\rangle_{4}= & \frac{1}{2}\left\{| \uparrow \rangle _ { e _ { 1 } } \left[\gamma_{1}\left|a_{1}\right\rangle\left(\delta_{1}\left|b_{1}\right\rangle+\delta_{2}\left|b_{2}\right\rangle\right)+\gamma_{2}\left|a_{2}\right\rangle\left(\delta_{2}\left|b_{1}\right\rangle\right.\right.\right. \\
& \left.\left.+\delta_{1}\left|b_{2}\right\rangle\right)\right]+|\downarrow\rangle_{e_{1}}\left[\gamma_{1}\left|a_{1}\right\rangle\left(\delta_{1}\left|b_{1}\right\rangle+\delta_{2}\left|b_{2}\right\rangle\right)\right. \\
& \left.\left.-\gamma_{2}\left|a_{2}\right\rangle\left(\delta_{2}\left|b_{1}\right\rangle+\delta_{1}\left|b_{2}\right\rangle\right)\right]\right\} \\
& \otimes\left\{| \uparrow \rangle _ { e _ { 2 } } \left[\alpha_{1}|R\rangle_{a}\left(\beta_{1}|R\rangle+\beta_{2}|L\rangle\right)_{b}+\alpha_{2}|L\rangle_{a}\right.\right. \\
& \left.\left(\beta_{2}|R\rangle+\beta_{1}|L\rangle\right)_{b}\right]+|\downarrow\rangle_{2}\left[\alpha _ { 1 } | R \rangle _ { a } \left(\beta_{1}|R\rangle\right.\right. \\
& \left.\left.\left.+\beta_{2}|L\rangle\right)_{b}-\alpha_{2}|L\rangle_{a}\left(\beta_{2}|R\rangle+\beta_{1}|L\rangle\right)_{b}\right]\right\} .
\end{aligned}
$$

By measuring the two excess electron spins $e_{1}$ and $e_{2}$ in the orthogonal basis $\{|\uparrow\rangle,|\downarrow\rangle\}$, a deterministic spatialpolarization hyper-CNOT gate can be constructed with feed-forward operations.

\section{(3) Feed-forward operations}

If an auxiliary photon with the initial state $\left|\varphi_{i}\right\rangle=$ $\frac{1}{\sqrt{2}}(|R\rangle+|L\rangle)$ is put into an optical microcavity, the state of the system composed of the photon and the electron spin after reflection is changed as follows:

$$
\begin{aligned}
& \frac{1}{\sqrt{2}}(|R\rangle+|L\rangle)|\uparrow\rangle \rightarrow \frac{1}{\sqrt{2}}(|R\rangle+i|L\rangle)|\uparrow\rangle, \\
& \frac{1}{\sqrt{2}}(|R\rangle+|L\rangle)|\downarrow\rangle \rightarrow \frac{1}{\sqrt{2}}(|R\rangle-i|L\rangle)|\downarrow\rangle .
\end{aligned}
$$

By detecting the auxiliary photon with the orthogonal linear polarization basis $\left\{\frac{1}{\sqrt{2}}(|R\rangle+i|L\rangle), \frac{1}{\sqrt{2}}(|R\rangle-i|L\rangle)\right\}$, the excess electron spin in $\mathrm{QD}$ can be read out. If the auxiliary photon is projected on $\frac{1}{\sqrt{2}}(|R\rangle+i|L\rangle)$, the excess electron spin is in the state $|\uparrow\rangle$. If the auxiliary photon is projected on $\frac{1}{\sqrt{2}}(|R\rangle-i|L\rangle)$, the excess electron spin is in the state $|\downarrow\rangle$. If we perform an addition sign change $\left|a_{2}\right\rangle \rightarrow-\left|a_{2}\right\rangle$ on the photon $a$ for $|\downarrow\rangle_{e_{1}}$ in $\mathrm{QD}_{1}$ and an addition sign change $|L\rangle_{a} \rightarrow-|L\rangle_{a}$ on the photon $a$ for $|\downarrow\rangle_{e_{2}}$ in $\mathrm{QD}_{2}$, the state of the system composed of the photons $a$ and $b$ becomes

$$
\begin{aligned}
\left|\Psi_{a b}\right\rangle_{c}= & {\left[\gamma_{1}\left|a_{1}\right\rangle\left(\delta_{1}\left|b_{1}\right\rangle+\delta_{2}\left|b_{2}\right\rangle\right)+\gamma_{2}\left|a_{2}\right\rangle\left(\delta_{1}\left|b_{2}\right\rangle\right.\right.} \\
& \left.\left.+\delta_{2}\left|b_{1}\right\rangle\right)\right] \otimes\left[\alpha_{1}|R\rangle_{a}\left(\beta_{1}|R\rangle+\beta_{2}|L\rangle\right)_{b}\right. \\
& \left.+\alpha_{2}|L\rangle_{a}\left(\beta_{1}|L\rangle+\beta_{2}|R\rangle\right)_{b}\right] .
\end{aligned}
$$

One can see that there is a bit flip on the spatial mode of the photon $b$ (the target qubit) when the spatial mode of the photon $a$ (the control qubit) is $\left|a_{2}\right\rangle$. Moreover, there is a bit flip on the polarization of the photon $b$ when the polarization of the photon $a$ is $|L\rangle_{a}$. That is, the outcome shown in Eq.(12) is the hyper-CNOT gate operating on the two-photon system on both its polarization and its spatial-mode DOFs. Moreover, this hyper-CNOT gate works in a deterministic way in principle. 

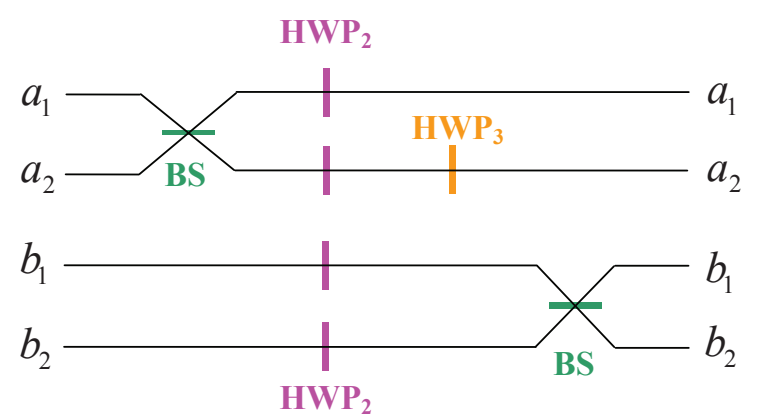

FIG. 3: (Color online) Schematic diagram for the preparation of a two-photon four-qubit cluster state with a hyperentangled Bell state. $\mathrm{HWP}_{2}$ is used to perform a polarization Hadamard operation. $\mathrm{HWP}_{3}$ represents a half-wave plate which is used to perform a polarization phase-flip operation $U_{p}=-|R\rangle\langle R|+$ $|L\rangle\langle L|$. BS represents beam splitter (50:50) which is used to perform a spatial mode Hadamard operation.

\section{Preparation of two-photon four-qubit cluster state}

As an application of our hyper-CNOT gate, we discuss the generation and the complete analysis of twophoton four-qubit cluster entangled states below. One can see that these tasks can be accomplished easily and simply with a hyper-CNOT gate and some linear-optical elements.

With our hyper-CNOT gate operating on the spatialmode and the polarization DOFs of a photon pair in the initial state $\frac{1}{2}\left[(|R\rangle+|L\rangle)_{a}\left(\left|a_{1}\right\rangle+\left|a_{2}\right\rangle\right) \otimes|R\rangle_{b}\left|b_{1}\right\rangle\right]$, it is, in principle, easily to prepare the hyperentangled twophoton four-qubit state

$\left|\Phi_{a b}\right\rangle_{1}=\frac{1}{2}\left(|R\rangle_{a}|R\rangle_{b}+|L\rangle_{a}|L\rangle_{b}\right) \otimes\left(\left|a_{1}\right\rangle\left|b_{1}\right\rangle+\left|a_{2}\right\rangle\left|b_{2}\right\rangle\right)$.

With linear optical elements, this hyperentangled state can be transformed into a two-photon four-qubit cluster state.

As shown in Fig 3 , after the Hadamard operations performed on both the polarization $\left(\mathrm{HWP}_{2}\right)$ and the spatialmode (BS) DOFs of the photon $a$, the hyperentangled Bell state becomes

$$
\begin{aligned}
\left|\Phi_{a b}\right\rangle_{2}= & \frac{1}{4}\left[(|R\rangle+|L\rangle)_{a}|R\rangle_{b}+(|R\rangle-|L\rangle)_{a}|L\rangle_{b}\right] \otimes \\
& {\left[\left(\left|a_{1}\right\rangle+\left|a_{2}\right\rangle\right)\left|b_{1}\right\rangle+\left(\left|a_{1}\right\rangle-\left|a_{2}\right\rangle\right)\left|b_{2}\right\rangle\right] . }
\end{aligned}
$$

Then we perform the spatial mode controlled polarization phase-flip gate $\left(\mathrm{HWP}_{3}\right)$ on the photon $a$, and the twophoton state $\left|\Phi_{a b}\right\rangle_{2}$ is transformed into $\left|\Phi_{a b}\right\rangle_{3}$. Here

$$
\begin{aligned}
\left|\Phi_{a b}\right\rangle_{3}= & \frac{1}{4}\left\{\left[(|R\rangle+|L\rangle)\left|a_{1}\right\rangle-(|R\rangle-|L\rangle)\left|a_{2}\right\rangle\right]_{a}|R\rangle_{b}\left|b_{1}\right\rangle\right. \\
& +\left[(|R\rangle+|L\rangle)\left|a_{1}\right\rangle+(|R\rangle-|L\rangle)\left|a_{2}\right\rangle\right]_{a}|R\rangle_{b}\left|b_{2}\right\rangle \\
& +\left[(|R\rangle-|L\rangle)\left|a_{1}\right\rangle-(|R\rangle+|L\rangle)\left|a_{2}\right\rangle\right]_{a}|L\rangle_{b}\left|b_{1}\right\rangle
\end{aligned}
$$

$$
\left.+\left[(|R\rangle-|L\rangle)\left|a_{1}\right\rangle+(|R\rangle+|L\rangle)\left|a_{2}\right\rangle\right]_{a}|L\rangle_{b}\left|b_{2}\right\rangle\right\} .
$$

After performing Hadamard operations on both the polarization $\left(\mathrm{HWP}_{2}\right)$ and the spatial-mode (BS) DOFs of the photon $b$ at last, we get the two-photon four-qubit cluster state

$$
\begin{aligned}
\left|\Phi_{a b}\right\rangle_{4}= & \frac{1}{2}\left[\left|a_{1}\right\rangle\left|b_{1}\right\rangle\left(|R\rangle_{a}|R\rangle_{b}+|L\rangle_{a}|L\rangle_{b}\right)\right. \\
& \left.-\left|a_{2}\right\rangle\left|b_{2}\right\rangle\left(|R\rangle_{a}|R\rangle_{b}-|L\rangle_{a}|L\rangle_{b}\right)\right] .
\end{aligned}
$$

From the process of the preparation of a two-photon four-qubit cluster state, one can see that this state can be disentangled to the hyperentangled Bell state $\left|\Phi_{a b}\right\rangle_{1}$ with some Hadamard operations and a spatial-mode controlled polarization phase-flip gate using linear optical elements. With a hyper-CNOT gate, the 16 hyperentangled Bell states can be transformed into 16 two-photon four-qubit product states which can be distinguished simply with linear optical elements and single-photon detectors. That is, the 16 hyperentangled Bell states can be analyzed easily with our spatial-polarization hyperCNOT gate.

\section{Discussion and Conclusion}

The spatial-polarization hyper-CNOT gate is constructed with the interaction of circularly polarized photons and one-side QD-cavity systems. According to Pauli's exclusion principle, this optical property is caused by the different reflection phase shifts of the left and the right circularly polarized photons. By adjusting the frequencies as $\omega_{c}=\omega_{X^{-}}=\omega_{0}, \omega-\omega_{c} \approx \kappa / 2$ and the cavity side leakage rate as $\kappa_{s}<1.3 \kappa[29]$, the relative phase shift of circularly polarized photons can achieve $\Delta \varphi=-\pi / 2$. Young et al 34] investigated the quantum-dot-induced phase shift experimentally in 2011, and showed that a QD-induced phase shift of $0.2 \mathrm{rad}$ between an (effectively) empty cavity $(Q \sim 51000, d=2.5 \mu \mathrm{m})$ and a cavity with a resonantly coupled QD can be deduced using a single-photon level probe. The Hadamard operation and unitary rotation operation of an electron spin can be completed by single-spin rotations using nanosecond electron spin resonance microwave pulses [35]. The electron spin coherence time can be extended to $\mu$ s using spin echo techniques [29] to protect the electron spin coherence with microwave pulses. The optical coherence time of an exciton is ten times longer than the cavity photon lifetime [36], with which the optical dephasing only reduces the fidelity a few percent. The hole spin dephasing is dominant in the spin dephasing of $X^{-}$, and it can be safely neglected with the hole spin coherence time three orders longer than the cavity photon lifetime [37]. The heavy-light hole mixing, which causes optical selection rule unperfect, could be reduced by engineering the shape, size and type of charged exciton [29]. 


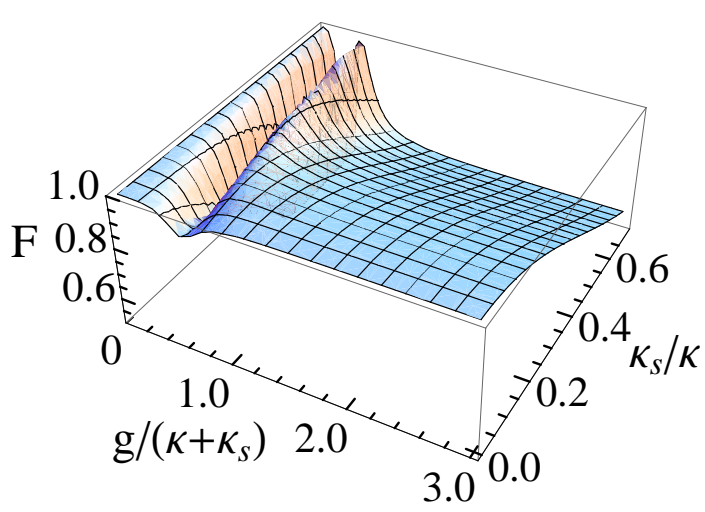

(a)

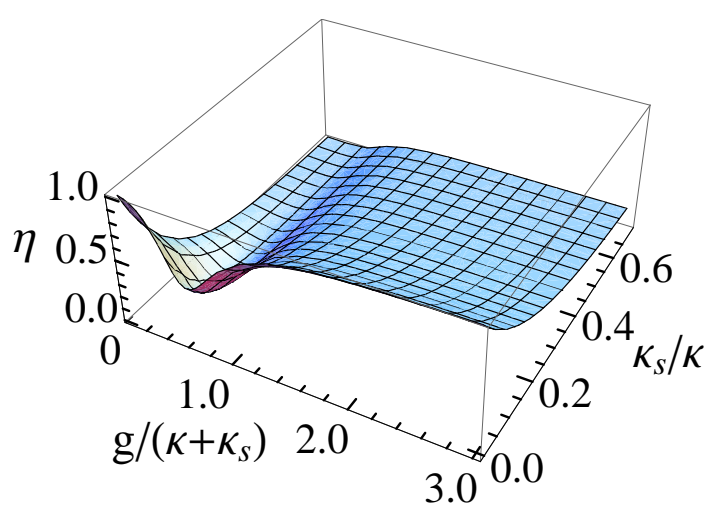

(b)

FIG. 4: (Color online) Fidelity and efficiency of spatialpolarization hyper-CNOT gate vs the coupling strength and side leakage rate with $\gamma=0.1 \kappa$.

In ideal conditions in which one neglects the cavity side leakage, the reflection coefficients are $\left|r_{0}(\omega)\right| \cong 1$ and $\left|r_{h}(\omega)\right| \cong 1$, and the fidelity of our spatial-polarization hyper-CNOT gate is nearly $100 \%$. Unfortunately, it is impossible to neglect side leakage from the cavity, this being a rigorous limitation to the QDCmicropillar cavity in the experiment, and the fidelity is reduced to $F=$ $\left|\left\langle\psi_{f} \mid \psi\right\rangle\right|^{2}$, where $|\psi\rangle$ and $\left|\psi_{f}\right\rangle$ describe the final states of an ideal condition and a whole process considering external reservoirs, respectively. The Faraday rotation angle is not very sensitive to the side leakage when $\kappa_{s}<\kappa$ [26]. Therefore, the fidelity $F$ and the efficiency $\eta$ of our spatial-polarization hyper-CNOT gate are

$$
\begin{aligned}
F= & \frac{\left[2\left(\left|r_{0}\right|+\left|r_{h}\right|\right)^{2}\right]^{4}}{\left[2\left(\left.|| r_{0}|+| r_{h}\right|^{2}+|| r_{0}|-| r_{h}||^{2}\right)\left(\left|r_{0}\right|^{2}+\left|r_{h}\right|^{2}\right)\right]^{2}} \\
& \times \frac{\left(\left|r_{0}\right|+\left|r_{h}\right|\right)^{4}}{\left(\left|r_{0}\right|^{2}+\left|r_{h}\right|^{2}\right)^{2}} \\
\eta= & {\left[\frac{1}{2}\left(\left|r_{0}\right|^{2}+\left|r_{h}\right|^{2}\right)\right]^{4} }
\end{aligned}
$$

The fidelity and the efficiency of our spatial-polarization
hyper-CNOT gate are shown in Fig. 4. One can see that this hyper-CNOT gate work efficiently in a strong coupling regime $\left(g>\left(\kappa+\kappa_{s}\right) / 4\right)$. It is challenging to achieve a strong coupling in QD-cavity systems experimentally. The coupling strength can be improved from $g \cong 0.5\left(\kappa+\kappa_{s}\right)$ (the quality factor $Q=8800$ ) [38] to $g \cong 2.4\left(\kappa+\kappa_{s}\right)(Q \sim 40000)$ [39] by engineering the sample designs, growth and fabrication in $d=1.5 \mu \mathrm{m}$ micropillar microcavities. If the coupling strength is $g \cong 0.5\left(\kappa+\kappa_{s}\right)$, we can get the fidelity and the efficiency as $F=94.3 \%$ and $\eta=48.9 \%$ for $\kappa_{s} / \kappa=0$. If the coupling strength is $g \cong 2.4\left(\kappa+\kappa_{s}\right)$, we can get the fidelity and the efficiency as $F=100 \%$ and $\eta=96.3 \%$ for $\kappa_{s} / \kappa=0$, and $F=94.7 \%$ and $\eta=47.3 \%$ for $\kappa_{s} / \kappa=0.2$. Both the fidelity and the efficiency become high with a strong coupling strength, but they are reduced by the side leakage of cavity.

In experiment, the quality factor $\mathrm{Q}$ is dominated by the side leakage and cavity loss rate in a micropillar, while $g$ is dominated by the trion oscillator strength and the cavity modal volume. $\mathrm{Hu}$ et al [29] pointed out that $\mathrm{Q}$ factor can be reduced by thinning down the top mirror which can increase $\kappa$ and keep $\kappa_{s}$ in constant. Therefore, the coupling strength can be reduced to $g \cong 1.3\left(\kappa+\kappa_{s}\right)$ in a high- $Q$ micropillar $(Q \sim 18900)$ with the side leakage rate set to be $\kappa_{s} / \kappa \sim 0.2$, and the corresponding fidelity and the efficiency are $F=96 \%$ and $\eta=42.3 \%$. It is quite demanded for high efficiency operations to observe a small $\kappa_{s} / \kappa$ in a strong coupling regime.

In conclusion, the construction of a deterministic spatial-polarization hyper-CNOT gate can be achieved with the giant optical Faraday rotation induced by a single-electron spin in a quantum dot inside a one-side optical microcavity as a result of cavity quantum electrodynamics. In order to obtain the giant optical Faraday rotation, the frequencies of input photon and cavity mode should be adjusted to $\omega-\omega_{c} \approx \kappa / 2$, and the side leakage and the cavity loss rate $\kappa_{s} / \kappa$ should be controlled as small as possible. With this hyper-CNOT gate, the preparation of two-photon four-qubit cluster states is easy, in principle, and the complete analysis of hyperentangled Bell states of two-photon systems is simple. We have analyzed the experimental feasibility of this hyper-CNOT gate, concluding that it can be implemented with current technology. This hyper-CNOT gate could give the powerful capability for quantum computing and quantum communication.

Certainly, we only discuss the construction of the spatial-polarization hyper-CNOT gate by exploiting the nonlinear optical property of one-side quantum dotcavity systems. It can be, in principle, constructed with other systems based on nonlinearity, such as cross-Kerr media, nitrogen-vacancy centers, wave-guide nanocavity, and so on. It is possible to construct multi-photon quantum logical gates in the same way, which means a scalable quantum computing based on two DOFs of photon systems is feasible. Moreover, the present hyper-CNOT gate can be used to create multi-photon hyperentangled 
states in more than one DOF and complete the analysis on these multi-photon hyperentangled states as well.
NECT-11-0031.

\section{ACKNOWLEDGEMENTS}

This work is supported by the National Natural Science Foundation of China under Grant No. 11174039 and

[1] Nielsen M A and Chuang I L 2000 Quantum Computation and Quantum Information, Cambridge University Press, Cambridge

[2] Schmidt-Kaler F, Häffner H, Riebe M, Gulde S, Lancaster G P T, Deuschle T, Becher C, Roos C F, Eschner $\mathrm{J}$ and Blatt R 2003 Nature (London) 422408

[3] Childs A M, Chuang I L and Leung D W 2001 Phys. Rev. A 64012314

Feng G R, Xu G F and Long G L 2013 Phys. Rev. Lett. 110190501

[4] Beenakker C W J, DiVincenzo D P, Emary C and Kindermann M 2004 Phys. Rev. Lett. 93020501

[5] Knill E, Laflamme R and Milburn G J 2001 Nature (London) 40946

[6] O'Brien J L, Pryde G J, White A G, Ralph T C and Branning D 2003 Nature (London) 426264

[7] Gasparoni S, Pan J W, Walther P, Rudolph T and Zeilinger A 2004 Phys. Rev. Lett. 93250502

[8] Kiesel N, Schmid C, Weber U, Ursin R and Weinfurter H 2005 Phys. Rev. Lett. 95210505

[9] Okamoto R, Hofmann H F, Takeuchi S and Sasaki K 2005 Phys. Rev. Lett. 95210506

[10] Nemoto K and Munro W J 2004 Phys. Rev. Lett. 93 250502

[11] Collins D, Gisin N, Linden N, Massar S and Popescu S 2002 Phys. Rev. Lett. 88040404

[12] Bose S, Knight P L, Plenio M B and Vedral V 1999 Phys. Rev. Lett. 835158

[13] Walther P, Resch K J, Rudolph T, Schenck E, Weinfurter H, Vedral V, Aspelmeyer M and Zeilinger A 2005 Nature (London) 434169

[14] Mair A, Vaziri A, Weihs G and Zeilinger A 2001 Nature (London) 412313

[15] Oemrawsingh S S R, Ma X, Voigt D, Aiello A, Eliel E R, 't Hooft G W and Woerdman J P 2005 Phys. Rev. Lett. 95240501

[16] Fiurášek J 2006 Phys. Rev. A 73062313

[17] Fiurášek J 2008 Phys. Rev. A 78032317

[18] Gong Y X, Guo G C and Ralph T C 2008 Phys. Rev. A 78012305

[19] Kwiat P G and Weinfurter H 1998 Phys. Rev. A 58 2623(R)

[20] Schuck C, Huber G, Kurtsiefer C and Weinfurter H 2006 Phys. Rev. Lett. 96190501

[21] Barreiro J T, Wei T C and Kwiat P G 2008 Nature Phys.

\section{2}

[22] Simon C and Pan J W 2002 Phys. Rev. Lett. 89257901

[23] Sheng Y B, Deng F G and Zhou H Y 2008 Phys. Rev. A 77042308

[24] Sheng Y B and Deng F G 2010 Phys. Rev. A 81032307 Sheng Y B and Deng F G 2010 Phys. Rev. A 82044305

[25] Sheng Y B, Deng F G and Long G L 2010 Phys. Rev. A 82032318

[26] Hu C Y, Young A, O'Brien J L, Munro W J and Rarity J G 2008 Phys. Rev. B $\mathbf{7 8} 085307$

[27] Hu C Y, Munro W J and Rarity J G 2008 Phys. Rev. B 78125318

[28] Hu C Y, Munro W J, O'Brien J L and Rarity J G 2009 Phys. Rev. B 80205326

[29] Hu C Y and Rarity J G 2011 Phys. Rev. B 83115303

[30] Bonato C, Haupt F, Oemrawsingh S S R, Gudat J, Ding D, van Exter M P and Bouwmeester D 2010 Phys. Rev. Lett. 104160503

[31] Warburton R J, Dürr C S, Karrai K, Kotthaus J P, Medeiros-Ribeiro G and Petroff P M 1997 Phys. Rev. Lett. 795282

[32] Hu C Y, Ossau W, Yakovlev D R, Landwehr G, Wojtowicz T, Karczewski G and Kossut J 1998 Phys. Rev. B 58 R1766

[33] Walls D F and Milburn G J 1994 Quantum Optics, Springer-Verlag, Berlin

[34] Young A B, Oulton R, Hu C Y, Thijssen A C T, Schneider C, Reitzenstein S, Kamp M, Höfling S, Worschech L, Forchel A and Rarity J G 2011 Phys. Rev. A 84011803

[35] Petta J R, Johnson A C, Taylor J M, Laird E A, Yacoby A, Lukin M D, Marcus C M, Hanson M P and Gossard A C 2005 Science 3092180

[36] Langbein W, Borri P, Woggon U, Stavarache V, Reuter D and Wieck A D 2004 Phys. Rev. B 70033301

[37] Brunner D, Gerardot B D, Dalgarno P A, Wüst G, Karrai K, Stoltz N G, Petroff P M and Warburton R J 2009 Science $\mathbf{3 2 5} 70$

[38] Reithmaier J P, Sȩk G, Löffler A, Hofmann C, Kuhn S, Reitzenstein S, Keldysh L V, Kulakovskii V D, Reinecke T L and Forchel A 2004 Nature (London) 432197

[39] Yoshie T, Scherer A, Hendrickson J, Khitrova G, Gibbs H M, Rupper G, Ell C, Shchekin O B and Deppe D G 2004 Nature (London) 432200 